\title{
Venta clandestina de rodenticidas, un problema de salud pública. Reporte de dos casos
}

\section{Clandestine sale of rodenticides, a health problem. Report of two cases}

Karla Isis Avilés-Martínez, ${ }^{1}$ José Carlos Villalobos-Lizardi, ${ }^{2}$ Adriana López-Enríquez ${ }^{3}$

\begin{abstract}
Resumen
ANTECEDENTES: La venta clandestina de rodenticidas es una manifestación de la pobreza y la exclusión social. Los rodenticidas adquiridos en estas circunstancias son accesibles porque tienen un canal de distribución eficiente. Su disponibilidad en casa, sin medidas de seguridad adecuadas y estrictas, representa un problema de salud potencialmente letal debido al contenido, falsificación, adulteración y etiquetado inadecuado o ausente. CASOS CLíNICOS: Se reportan dos casos clínicos, no letales, de niños previamente sanos que ingirieron rodenticidas no etiquetados y obtenidos de la venta ilegal ambulante. El primer caso sufrió intoxicación por difetaliona (Clase la), rodenticida anticoagulante de segunda generación (en la bibliografía se reporta intoxicación en dos niños). El segundo caso tuvo intoxicación por carbofuran (Clase lb) y sufrió un toxíndrome colinérgico (existen limitados reportes bibliográficos en niños).

CONCLUSIONES: Los significativos avances en medicina y las herramientas de diagnóstico y tratamiento son siempre complementarias a la responsabilidad social del Pediatra en servicios deUrgencias; le corresponde reconocer el problema clínico y social, advertir a la población de los riesgos mortales de los productos adquiridos por venta clandestina y los mecanismos para prevenirlos.

PALABRAS CLAVE: Rodenticidas; niños; difetaliona; carbofuran; factores socieconómicos; servicios de Urgencias; hospital; agentes colinérgicos.
\end{abstract}

Abstract

BACKGROUND: The clandestine sale of chemical substances, such as rodenticides, is a manifestation of poverty and social exclusion. It reflects the effects of economic inequality, which, in turn, establishes consumption patterns. The rodenticides acquired in these circumstances are accessible, have an efficient distribution channel, the sellers sponsor them effectively and their price is low. On the other hand, they imply a potentially lethal health problem due to content, falsification, adulteration and inadequate or absent labeling. This manuscript places in perspective the complex work of the pediatrician before scenarios of this magnitude.

CLINICAL CASES: Two non-lethal clinical cases are presented in previously healthy children who ingested unlabeled rodenticidal products obtained from illegal street vendors; the first case with intoxication due to difethialone, second-generation anticoagulant rodenticide (intoxication is reported in 2 children in the literature). The second case presented intoxication by carbofuran and developed a cholinergic toxin syndrome (there are limited bibliographic reports in children). Tools to make diagnostic and therapeutic decisions based on the available literature are shown.

CONCLUSIONS: From this work it is concluded that the significant advances in medicine and the tools of diagnosis and treatment are always complementary to the social responsibility of the Pediatrician in the Emergency Department; It corresponds to recognize the clinical and social problem, warn the population about the lethal risks of the products acquired by clandestine sale and the mechanisms to prevent them.

KEY WORDS: Rodenticides; Child; Difethialone; Carbofuran; Socioeconomic factors; Emergency service, Hospital; Cholinergic agents.

\footnotetext{
${ }_{1}^{1}$ Pediatra adscrita al servicio de Urgencias pediátricas.

${ }^{2}$ Residente de segundo año de la especialidad en Pediatría médica.

${ }^{3}$ Pediatra urgencióloga, jefa del servicio Urgencias Pediátricas.
}

Hospital Civil Fray Antonio Alcalde, Guadalajara, Jalisco, México

Recibido: 21 de enero 2018

Aceptado: 8 de febrero 2019

Correspondencia

Karla Isis Avilés Martínez

draisispediatra@yahoo.com.mx

Este artículo debe citarse como

Avilés-Martínez KI, Villalobos-Lizardi JC, López-Enríquez A. Venta clandestina de rodenticidas, un problema de salud pública. Reporte de dos casos. Acta Pediatr Mex. 2019;40(2):71-84. 


\section{ANTECEDENTES}

Puesto que los plaguicidas no están diseñados para consumo humano, los casos de envenenamiento en niños pueden ser dramáticos y difíciles de reconocer. La oralidad de los niños, su mimetismo con el medio, su curiosidad y peculiaridades fisiológicas, los hace particularmente vulnerables ante las repercusiones de una intoxicación. Las posibilidades de intoxicarse se incrementan ante sustancias químicas tóxicas accesibles en contenedores improvisados y no etiquetados (o con datos insuficientes o inciertos en la etiqueta). Este fenómeno es más frecuente en niños que se intoxican con plaguicidas y rodenticidas obtenidos de la venta clandestina ${ }^{1,2}$ o por negligencia doméstica, o ambas. ${ }^{3}$ En estas circunstancias aumenta el grado de dificultad para el clínico en urgencias, pues es imprescindible la correcta e inmediata identificación de la(s) sustancia(s) que causa la intoxicación para comprender la complejidad fisiopatológica, la potencial letalidad del tóxico y tomar las mejores y oportunas decisiones terapéuticas. Por lo tanto, las intoxicaciones por rodenticidas obtenidos de la venta clandestina son un problema de salud en todo el mundo. ${ }^{4}$

Las intoxicaciones por plaguicidas comprenden a los insecticidas-acaricidas, herbicidas, molusquicidas, fungicidas y rodenticidas. Tienen en común las vías de absorción: inhalación, ingesta (accidental o no) y absorción cutánea. ${ }^{5,6}$ Los niños menores de cinco años sufren, aproximadamente, $3.3 \%$ de las intoxicaciones. ${ }^{7}$ Las intoxicaciones por rodenticidas, insecticidas y herbicidas se han reportado como causa de muerte en $17-19 \%$ de los casos. ${ }^{8}$ En la actualidad se dispone de bibliografía limitada acerca de la prevalencia de exposiciones a plaguicidas en niños; esto repercute en la escasa disponibilidad de evidencia de su tratamiento; por lo tanto, el tratamiento actual se sustenta en consensos de juicios de las mejores opciones disponibles.
La Organización Mundial de la Salud (OMS) clasifica a los plaguicidas, sobre todo, con base en su toxicidad aguda en estudios efectuados en animales. Se clasifican en extremadamente peligrosos (Ia), altamente peligrosos (Ib), moderadamente peligrosos (II), poco peligrosos (III), normalmente no ofrecen peligro mediante uso adecuado (U); fumigante no clasificado (FM) y pesticida obsoleto, no clasificado $(\mathbf{O})$ (The WHO Recommended Classification of Pesticides by Hazard and Guidelines to Classification 2009).

Se han descrito cinco toxíndromes cuya presentación puede ser clásica o tener formas incompletas: anticolinérgico, colinérgico, simpaticomimético, hipnótico-sedante y opioide como herramienta diagnóstica en el abordaje general en urgencias de los niños con intoxicación por alguna sustancia química. Estos toxíndromes están integrados por anormalidad en 10 parámetros clínicos: signos vitales (frecuencia cardiaca, frecuencia respiratoria, presión arterial, temperatura), evidencias neurológicas (diámetro pupilar y reactividad), estado neurológico (agitación, sedación, convulsiones, coma), piel (color, humedad), mucosas (húmedas, secas), actividad gastrointestinal (ileo, peristaltismo incrementado), manifestaciones urinarias (retención, incontinencia). ${ }^{9}$ Cuadro 1

\section{Caso 1}

Paciente pediátrico de 1 año, referido sano; fue llevado en tres ocasiones previas al hospital por urgencias sentidas. Vive a unas cuadras del hospital con su madre y hermana en alojamiento de una habitación. En esta ocasión, la madre lo encontró comiendo 3-4 cubos rojos de veneno para ratas que compró (sin etiquetas) en un mercado local. Minutos más tarde ingresó a triage, en donde se le observó Iloroso, consolable con la madre, sin incremento del esfuerzo respiratorio, coloración de piel rosada. Frecuencia cardiaca 130 latidos por minuto, frecuencia respiratoria 25 respiraciones por minuto, temperatura $37^{\circ} \mathrm{C}$, pupilas 
Cuadro 1. Clasificación de los plaguicidas según su toxicidad en animales

\begin{tabular}{|c|c|c|c|c|c|c|}
\hline \multirow[t]{2}{*}{ Clasificación según los riesgos (OMS) } & \multirow{2}{*}{$\begin{array}{c}\text { Clasificación } \\
\text { de peligro }\end{array}$} & \multirow{2}{*}{\multicolumn{2}{|c|}{$\begin{array}{l}\text { Color de la } \\
\text { banda }\end{array}$}} & \multirow[t]{2}{*}{ leyenda } & \multicolumn{2}{|c|}{$\mathrm{DL}_{50}$ para ratas $/ \mathrm{kg}$} \\
\hline & & & & & Oral & Dérmico \\
\hline $\begin{array}{l}\text { Clase (la) producto } \\
\text { sumamente tóxico }\end{array}$ & Muy tóxico & Rojo & $\square$ & Muy tóxico & $<5$ & $<50$ \\
\hline Clase (Ib) producto muy peligroso & Tóxico & Rojo & ש & Tóxico & $5-50$ & $50-200$ \\
\hline Clase (II) producto moderádamente peligroso & Nocivo & Amarillo & $\square$ & Nocivo & $50-2000$ & $200-2000$ \\
\hline Clase (III) producto poco peligroso & Cuidado & Azul & $\square$ & Cuidado & $>2000$ & $>2000$ \\
\hline Clase $(U)$ producto que no ofrece riesgos & & Verde & $\square$ & Cuidado & \multicolumn{2}{|c|}{5000 o más } \\
\hline Clase (O) obsoleto como pesticida. No clasificado & & Naranja & $\square$ & & & \\
\hline
\end{tabular}

Referencias: 1-3,8-25

isocóricas, saturación de oxígeno ambiente 98\%, peso $8.3 \mathrm{~kg}$, talla $70 \mathrm{~cm}$. Se ingresó en Urgencias por la ingesta de posible rodenticida.

En la evaluación primaria (signos vitales, permeabilidad de vía aérea, respiración, circulación, estado neurológico, exposición general) no se documentaron anormalidades. En la evaluación secundaria (evaluación instrumentada y completa de cabeza a pies) se encontró exantema máculo-papular en el tronco y las extremidades, sin otro dato agregado. Se integró rápidamente su historia clínica, SAMPLE por su acrónimo; signos y síntomas: negados; alergias: desconocidas; medicamentos: ninguno; historia médica previa: madre de 20 años, con apendicectomía por apendicitis complicada con perforación en la semana 32 de gestación (embarazo gemelar), la hermana del niño tiene microcefalia y síndrome de West, los dos niños estuvieron hospitalizados durante cinco semanas después del nacimiento; esquema de vacunación incompleto; ingesta de último alimento aproximadamente 2 horas previas, evento: ingesta de rodenticida. Se le interrogó acerca de potenciales sustancias tóxicas en el domicilio, mencionó que existen medicamentos de la hermana del niño en un botiquín y fuera del alcance. Se identificó como clínicamente estable, más ante la ingesta de una sustancia tóxica, se realizó descontaminación cutánea y lavado gástrico de donde se obtuvieron 4 cubos del tóxico y residuo alimenticio, se inició la administración de $1 \mathrm{~g} / \mathrm{kg}$ de carbón activado y $1 \mathrm{~g} / \mathrm{kg}$ de manitol oral.

Se le realizaron estudios complementarios: electrocardiograma, tiempos de coagulación y biometría hemática. Como estrategia para la identificación del tóxico se buscaron imágenes en internet compatibles con las características macroscópicas del probable rodenticida, que se identificó como difetialona (familia hidroxi4-benzotiapiranonas). Se le administraron $2 \mathrm{mg}$ de fitonadiona subcutánea (Cuadros 2 y 3). El niño permaneció estable, y en revisión de tiempos de coagulación por al menos 24 horas. Los resultados iniciales fueron normales; a las 12 horas se encontró el tiempo de protrombina en 25", que permaneció elevado durante 18 horas, sin evidencia de sangrado. Se continuó con fitonadiona.

Se dio de alta del hospital a las 72 horas, asintomático. Dos semanas después, en la cita de control, la exploración física y los estudios de laboratorio fueron normales. Se decidió el alta definitiva y se indicaron a la madre medidas higiénico-dietéticas, de cuidado y prevención de 


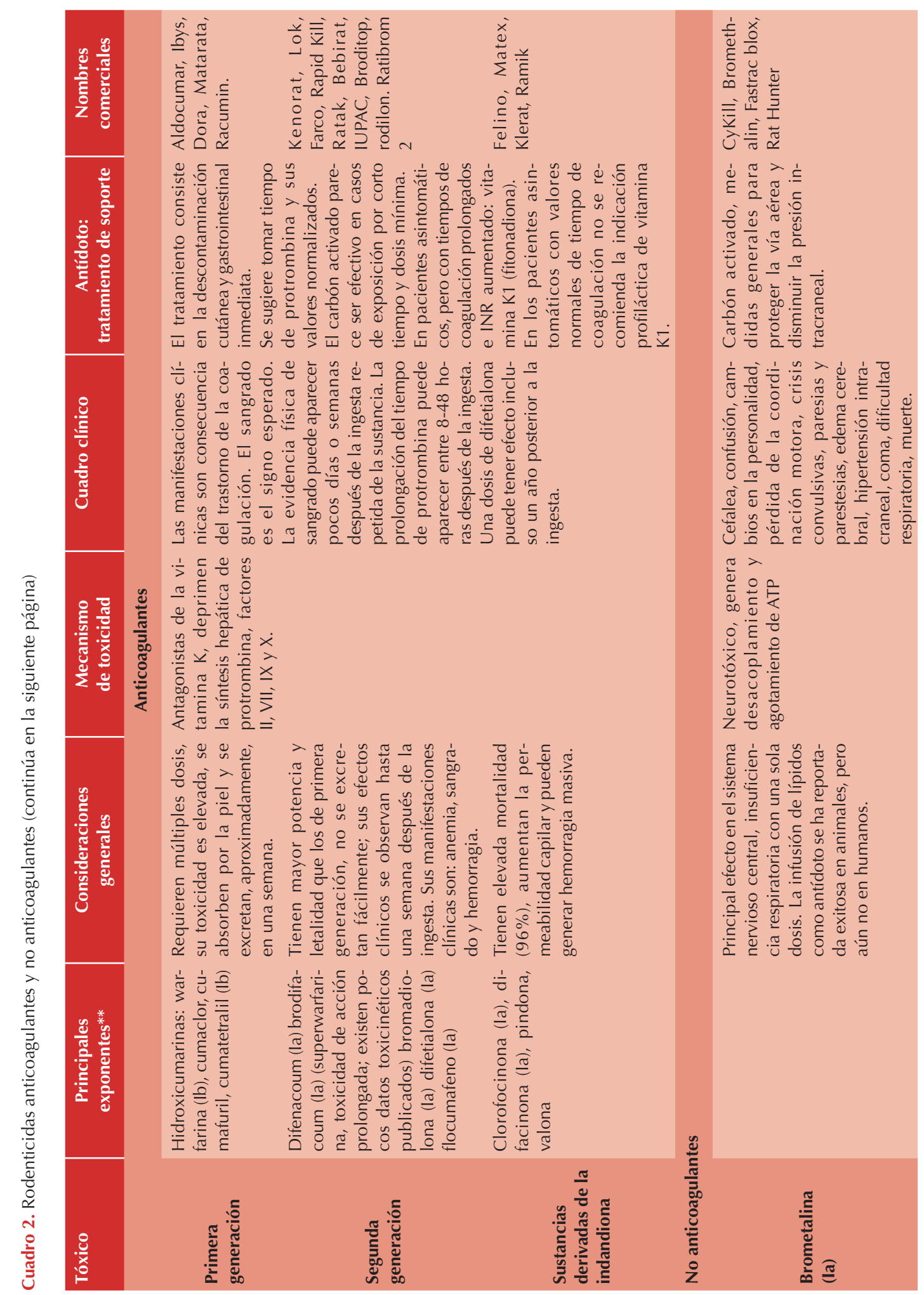




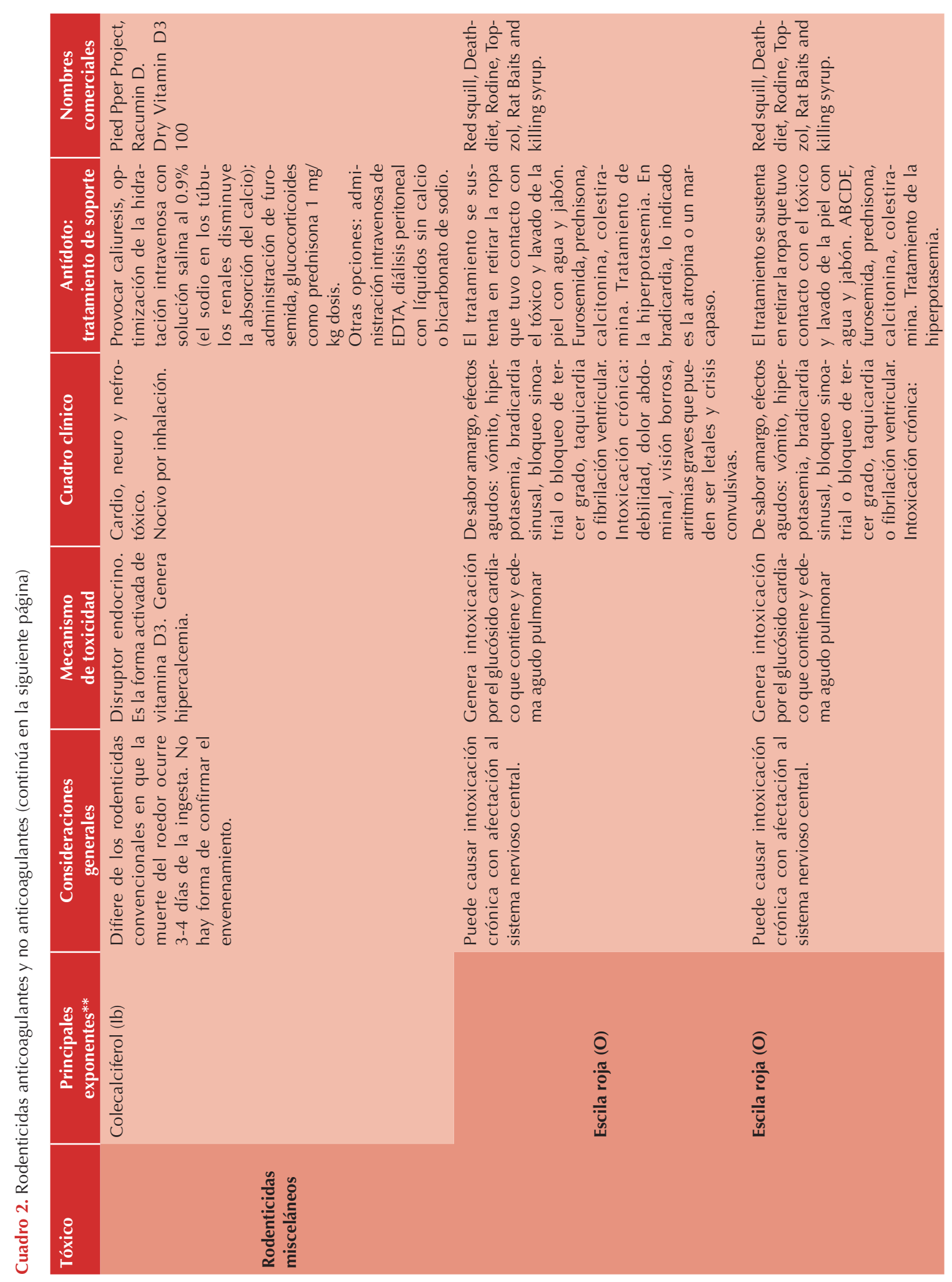




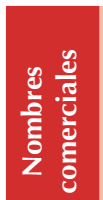

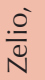

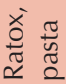

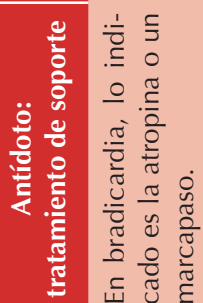

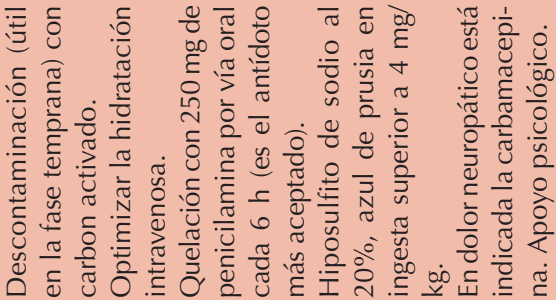

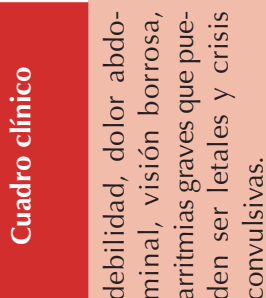

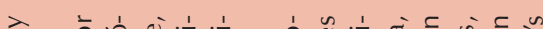

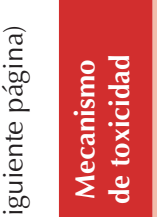

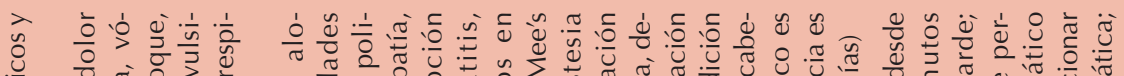

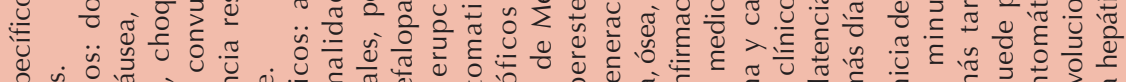

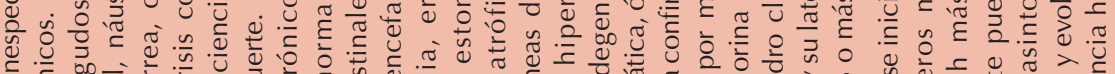

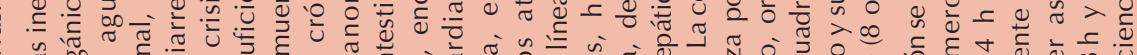

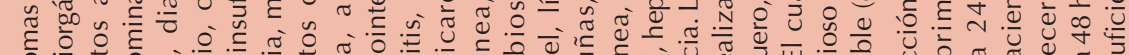

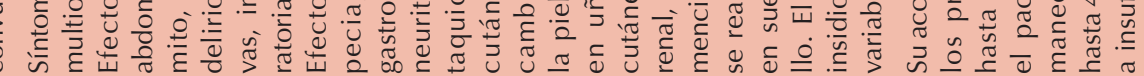
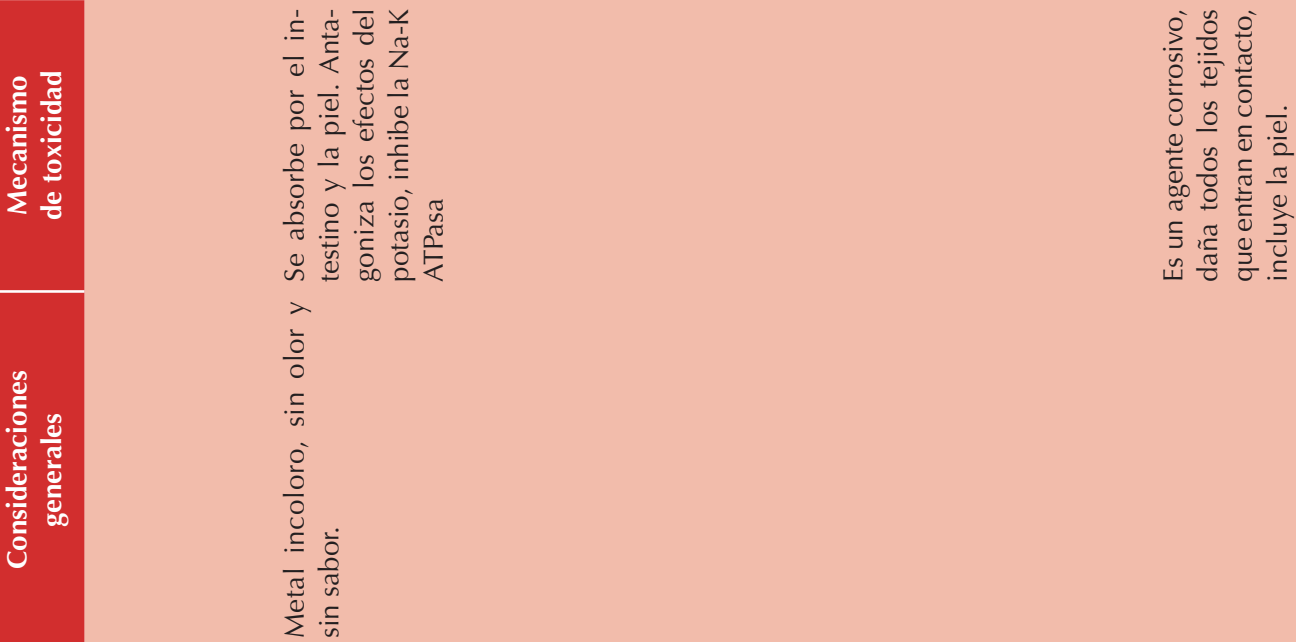

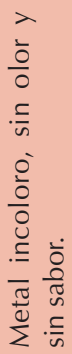




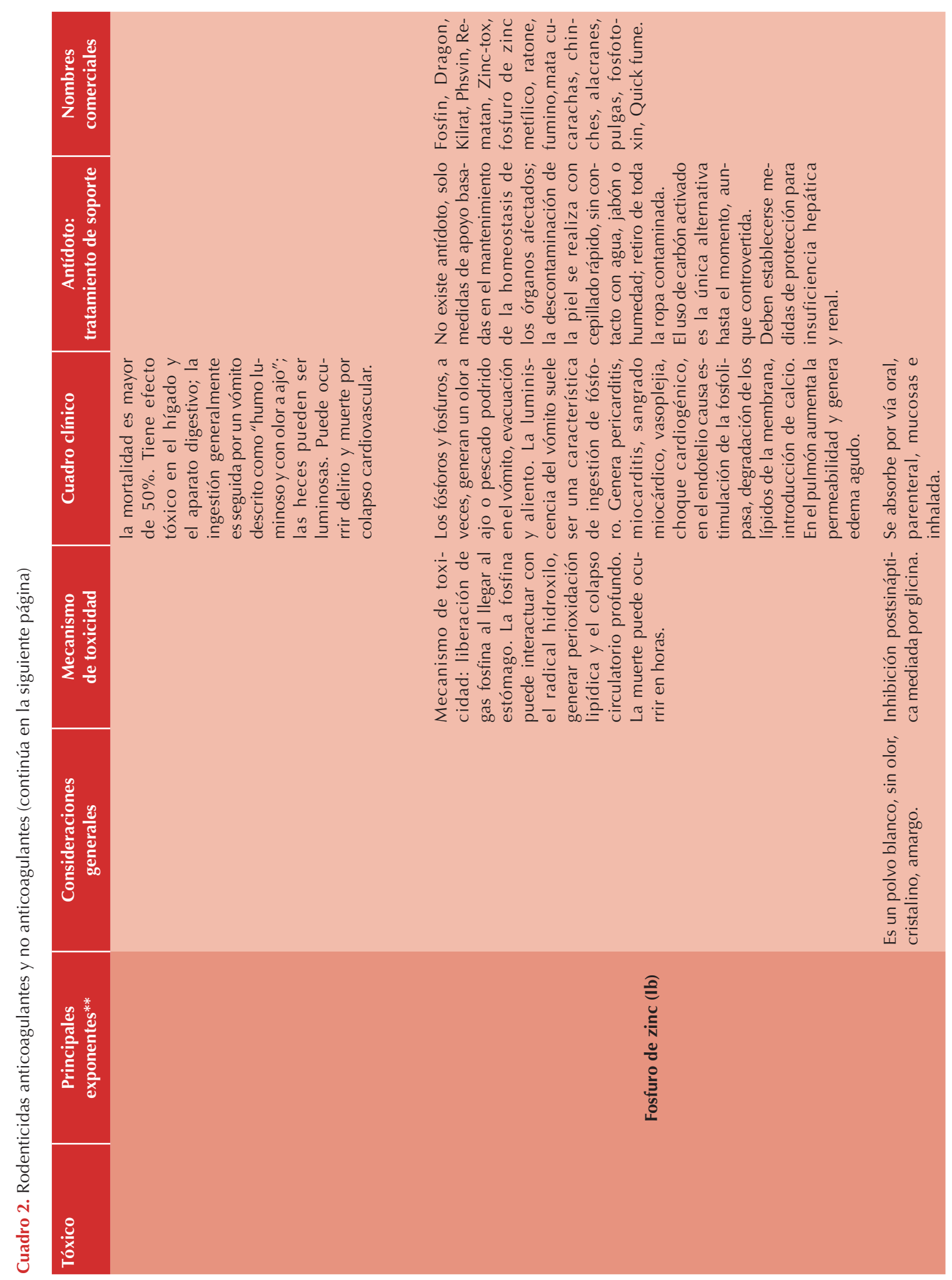




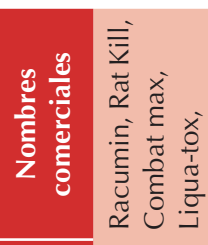

ఏँ

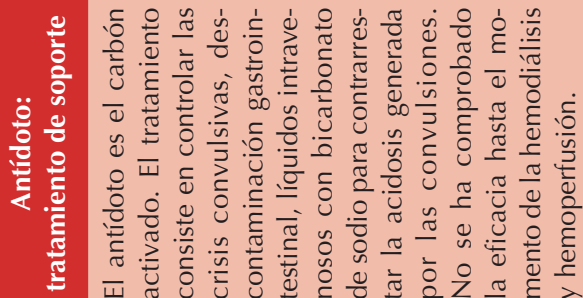

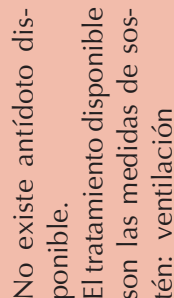

ฮึ

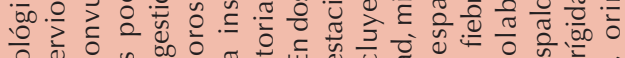

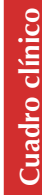

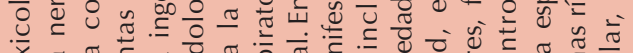

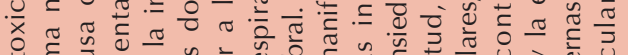

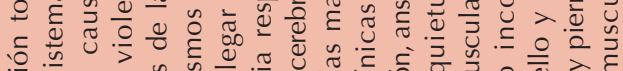

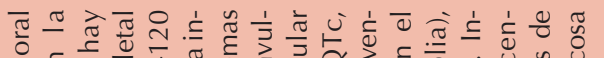

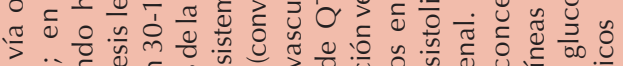

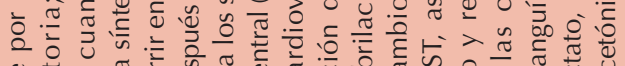

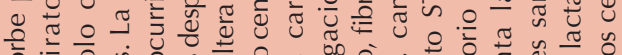

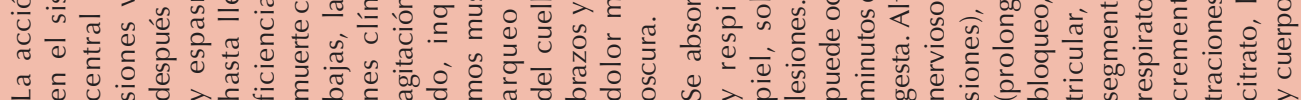

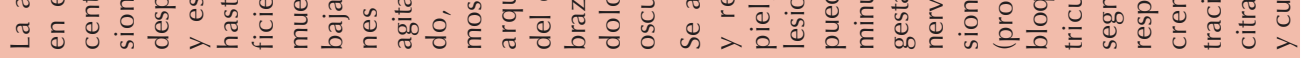

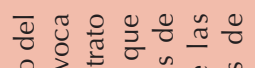

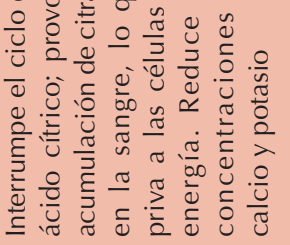

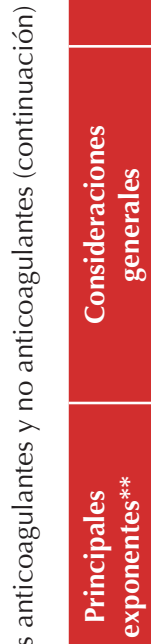

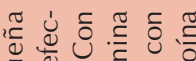

守.

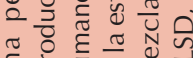

胥

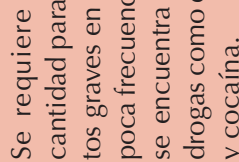

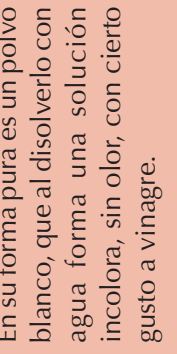

는

쥬요

ह. 웡 巳

U. 융

는 원

응

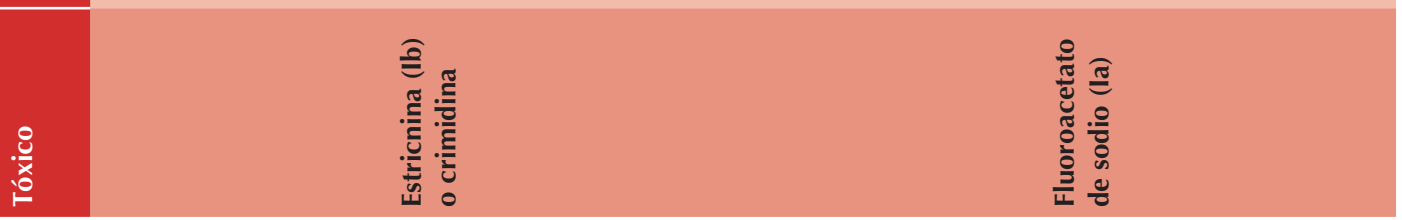

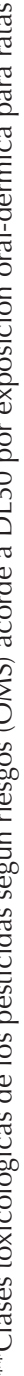


Cuadro 3. Comparación de signos y síntomas del caso clínico intoxicado por difetialona

\begin{tabular}{|l|c|}
\hline Signos y síntomas & $\begin{array}{c}\text { Caso comparado } \\
\text { con } 5 \text { estudios (10 } \\
\text { pacientes) }\end{array}$ \\
\hline Niños e infantes & $\checkmark$ \\
\hline Ingesta accidental & $\checkmark$ \\
\hline Inicialmente asintomático & $\checkmark$ \\
\hline Hematuria & $\mathrm{X}$ \\
\hline Gingivorragia & $\mathrm{X}$ \\
\hline Epistaxis & $\mathrm{X}$ \\
\hline Otorragia & $\mathrm{X}$ \\
\hline Dolor lumbar & $\mathrm{X}$ \\
\hline Hematoma abdominal & $\mathrm{X}$ \\
\hline Hematemesis & $\mathrm{X}$ \\
\hline Melenas & $\mathrm{X}$ \\
\hline Trombosis & $\mathrm{X}$ \\
\hline Infarto de miocardio & $\mathrm{X}$ \\
\hline Coma & $\mathrm{X}$ \\
\hline TP prolongado & $\checkmark$ \\
\hline TPT prolongado & $\mathrm{X}$ \\
\hline Tratamiento fitonadiona & $\checkmark$ \\
\hline Tratamiento carbón activado & $\mathrm{X}$ \\
\hline Hemartrosis & $\checkmark$ \\
\hline Muerte & \\
\hline & \\
\hline
\end{tabular}

Referencias 26-30

accidentes en el hogar y el Comité Hospitalario de Protección al menor continuó el seguimiento.

\section{Caso 2}

Paciente masculino, de cinco años, Ilevado al servicio de Atención Médica de Urgencias. Radica cerca de un aserradero, en un municipio rural. La madre refirió que ese día, temprano por la mañana, colocó veneno para ratas en unos bolillos sobre los roperos. El veneno (Iíquido rosa) lo obtuvo sin etiqueta de familiares, quienes le comentaron que era para ardillas y otros roedores. A media tarde, la madre encontró al niño comiendo el pan con el tóxico; lo hizo ingerir agua y leche, tuvo mareo, náuseas y arcadas pospandriales inmediatas. Una hora más tarde, la madré lo llevó a la Unidad de Protección Civil de la localidad, donde el niño ingresó somnoliento, pálido, tono muscular disminuido y mucosas secas. Fue trasladado en ambulancia a un hospital de primer contacto, tratado con oxígeno en puntas nasales. En la quinta hora de evolución tuvo insuficiencia respiratoria, que requirió intubación endotraqueal y ventilación asistida. Le administraron furosemida, corticoide y difenhidramina. Una hora después lo trasladaron al Servicio de Urgencias del hospital para atención definitiva.

En la evaluación inicial se documentó su estado neurológico: movimientos anormales, respiratorio: incremento del trabajo respiratorio, disociado de la ventilación asistida; circulatorio: pálido. En la evaluación primaria se obtuvieron signos vitales: frecuencia cardiaca irregular 40-60 latidos por minuto, temperatura $36{ }^{\circ} \mathrm{C}$, ventilación asistida 20 ciclos por minuto, presión arterial 112-70 $\mathrm{mmHg}$, saturación de oxígeno $100 \%$, peso $20 \mathrm{~kg}$. Vía aérea no permeable, broncorrea profusa, Ilenado capilar de 3 segundos, pupilas mióticas puntiformes, arrefléxicas, sin respuesta fotoluminosa, sin datos agregados en la exposición. Se integró insuficiencia cardiorrespiratoria y un toxíndrome colinérgico.

La primera intervención urgente consistió en aspirar secreciones y administración de una dosis de benzodiacepina para control y sincronía con la ventilación mecánica. En la evaluación secundaria se completó SAMPLE: signos de insuficiencia respiratoria, hipotonía muscular y choque, alergias negadas, esquema de vacunación completo, sin medicamentos diferentes a los descritos $u$ otras sustancias tóxicas en el domicilio, referido sano previamente; último alimento 6 horas previas; el motivo de traslado fue el deterioro neurológico, respiratorio y circulatorio. Se le administraron $0.02 \mathrm{mg} / \mathrm{kg} /$ dosis de atropina, ante la persistencia de la bradiarritmia 
se le administró una segunda dosis. La arritmia revirtió con la cuarta dosis del fármaco. Se inició la administración de carbón activado mediante sonda orogástrica, no se agregó catártico.

El niño tuvo una evacuación diarreica, fétida, abundante y se trasladó inmediato al área de decontaminación que cuenta con campana de extracción. Como parte de la evaluación complementaria se tomaron estudios de laboratorio: gasometría arterial con acidosis metabólica compensada inicial (brecha aniónica 21), hipopotasemia de $2.6 \mathrm{mEq} / \mathrm{L}$, tiempo de protrombina $16^{\prime \prime}$, tiempo parcial de tromboplastina 28". Se repuso el potasio y se logró la normalización del ritmo cardiaco. Seis horas más tarde las pupilas eran isocóricas, normorrefléxicas, disminución del gasto fecal y su fetidez. Las concentraciones de colinesterasa eritrocitaria se reportaron en $4 \mathrm{U} / \mathrm{mL}$ (valor de referencia $8-13 \mathrm{U} / \mathrm{mL}$ ) ante los datos clínicos de toxíndrome colinérgico. Doce horas después del ingreso, los servicios de protección civil identificaron el tóxico como carbofuran (2,3-dihidro-2,2-dimetil-7-benzofuranil-metilcarbamato) (Cuadro 4). Luego de

Cuadro 4. Comparación de signos y síntomas por carbofuran en el caso clínico versus la bibliografía disponible (continúa en la siguiente columna)

\begin{tabular}{|l|c|}
\hline Signos y síntomas & $\begin{array}{c}\text { Caso comparado con } \mathbf{2 8} \\
\text { estudios (890 pacientes) }\end{array}$ \\
\hline Niños & $\checkmark$ \\
\hline Intoxicación aguda & $\checkmark$ \\
\hline Intoxicación crónica & $X$ \\
\hline Accidental/ocupacional & $\checkmark$ \\
\hline Muerte & $X$ \\
\hline Toxíndrome colinérgico & $\checkmark$ \\
\hline Signo muscarínico & $\checkmark$ \\
\hline Miosis & $\checkmark$ \\
\hline Diaforesis & $X$ \\
\hline Sialorrea & $\checkmark$ \\
\hline Dolor torácico & $X$ \\
\hline Disnea & $X$ \\
\hline
\end{tabular}

Cuadro 4. Comparación de signos y síntomas por carbofuran en el caso clínico versus la bibliografía disponible (continúa en la siguiente página)

\begin{tabular}{|l|c|}
\hline Signos y síntomas & $\begin{array}{c}\text { Caso comparado con } \mathbf{2 8} \\
\text { estudios }\end{array}$ \\
\hline Insuf pacientes)
\end{tabular}


Cuadro 4. Comparación de signos y síntomas por carbofuran en el caso clínico versus la bibliografía disponible (continuación)

\begin{tabular}{l|c|}
\hline Signos y síntomas & $\begin{array}{c}\text { Caso comparado con } \mathbf{2 8} \\
\text { estudios }\end{array}$ \\
\hline Lesión renal & Xacientes)
\end{tabular}

Referencias: 4,7,18,31-54

trascurridas 24 horas estable se trasladó aa la unidad de cuidados intensivos pediátricos; 20 horas después, se logró el destete ventilatorio. Al sexto día fue dado de alta del hospital, asintomático.

En los casos descritos, sin más información del potencial tóxico, surgieron varias preguntas: ¿qué tipo de rodenticida originó el cuadro clínico?, ¿en cuánto tiempo aparecerán las primeras manifestaciones de toxicidad y cuáles son?, ¿qué tan letal es?, ¿cuál es el tratamiento y qué medidas de seguridad debían observarse?, ¿cuál es la responsabilidad social del médico urgenciólogo para evitar eventos como los descritos?

\section{DISCUSIÓN}

Muchos aspectos de la fisiología de los roedores y humanos son similares. Como tal, los agentes que se usan para envenener ratas y ratones son tóxicos para los humanos. Por esta razón, los rodenticidas ocupan un lugar destacado en la historia de la Medicina y de la Toxicología. ${ }^{16}$ Pocas consultas generan tanta angustia en las familias y tanta inquietud en los profesionales de la salud como la que propicia el niño con una potencial o real intoxicación..$^{3,9}$

Los factores que propician la venta clandestina de rodenticidas son múltiples y heterogéneos. El elemento común es la pobreza y la exclusión social que obliga al trabajo informal como la venta ambulante, legal o ilegal. Además, la desigualdad económica en la población de menor poder adquisitivo origina patrones particulares de consumo que incluyen las compras de dudosa legalidad. A esto se suman una educación médica insuficiente hacia la comunidad, el desconocimiento de riesgos de quienes venden, compran y usan estos productos, la imperfecta legislación y la escasa responsabilidad social.

Es común que la venta ocurra ante la vista pública. Un vendedor ambulante motivado hace del rodenticida clandestino un producto accesible, tiene un canal de distribución eficiente y su precio es bajo, incluso puede contar con la recomendación de boca en boca por otros miembros de la comunidad. En contraparte, implican un problema de salud grave y potencialmente letal, debido a la falsificación, adulteración y etiquetado inadecuado o ausente.

La magnitud del problema derivado de la venta clandestina de rodenticidas afecta a gran parte de las naciones del mundo; incluso, posterior a la implementación de rigurosas medidas de seguridad, así como imposicion de graves sanciones a corporaciones y a sus funcionarios, persiste la venta de estos productos que además involucran daños ambientales y actos criminales. El problema se extiende, pues actualmente no se limita a la venta ambulante, también pueden 
adquirirse algunas de estas sustancias químicas en ciertos negocios establecidos. ${ }^{1,2,4,7,16,17}$ Las principales causas de intoxicación en humanos por rodenticidas de venta clandestina están relacionadas, principalmente, con los intentos de suicidio, de homicidio y la intoxicación no intencional, especialmente algunos trabajadores del campo y en niños. ${ }^{4}$

Las sustancias químicas camufladas, fuera de los envases originales, sin etiquetas informativas o con información ilegible o incierta, ponen en mayor riesgo de muerte a los niños que ingieren estos productos y esto disminuye la oportunidad del clínico de identificar y tratar oportuna y eficazmente al niño intoxicado.

En abril de 2018 se llevó a cabo el Primer Foro Nacional sobre Plaguicidas en México, en conjunto con la Organización Panamericana de la Salud (OPS), la Organización Mundial de la Salud (OMS), Organización de Naciones Unidas Medio Ambiente (ONU), en coordinación con la Secretaría del Medio Ambiente y Recursos Naturales (SEMARNAT) y el Instituto Nacional de Ecología y Cambio Climático (INECC), en donde se insistió en que: "El uso de plaguicidas representa un importante riesgo para la salud y se puede prevenir a través de acciones conjuntas de diferentes actores, buscando siempre proteger a la población rural y urbana" (Gerry Eijkemans).

La importancia en materia de salud pública también la ha hecho palpable el Secretariado Técnico de Consejo Nacional para Prevención de Accidentes (CONAPRA) que incluye en el Modelo Integral de Prevención de Accidentes en grupos vulnerables en México, los envenenamientos e intoxicaciones. Para que en México un producto rodenticida reciba la aprobación de la agencia sanitaria (COFEPRIS y CICOPLAFEST), se analizan diversos elementos: riesgos, uso y la categoría. Respecto a la evaluación de los riesgos, la toxicidad de la sustancia y sus concentraciones en el producto, el propósito y las condiciones de uso, la ocurrencia de eventos adversos, quejas técnicas previas, las poblaciones expuestas probables, la frecuencia de exposición y de duración y las formas de preparación. Además, las compañías legalmente autorizadas para fabricar, almacenar, distribuir, transportar, fraccionar o importar productos rodenticidas están sujetas a la verificación del cumplimiento de las buenas prácticas de manufactura y control. Pese a que no se cuenta con reportes nacionales oficiales, en particular de difetaliona, como se menciona en el Catálogo Oficial de Plaguicidas, no debería desestimarse su clasificación como potencialmente peligroso (Clasificación la, $\mathrm{DL}_{50} 0.56 \mathrm{mg} / \mathrm{kg}$ ). Al carbofuran se le concede categoría letal y existe autorización para su uso como rodenticida.

Se han identificado diversas formulaciones ilegales que contienen inhibidores de colinesterasa, incluidas las formas sólidas que simulan anticoagulantes rodenticidas en forma de cebo y preparaciones líquidas, con etiquetas falsas y otros nombres. Las etiquetas de estos productos, a menudo, identifican a las cumarinas como ingredientes activos; sin embargo, pueden contener otros ingredientes, como carbofuran y organofosforados. Los hallazgos de diversos estudios indican que el envenenamiento por rodenticidas ilegales, que contienen inhibidores de acetilcolinesterasa, es generalmente grave, incluidos el carbofuran y la mayoría de los pacientes resulta con síndrome colinérgico, con predominio de manifestaciones muscarínicas. ${ }^{4,16}$ Los pesticidas constituyen un grupo extraordinariamente heterogéneo de sustancias químicas.

El carbofuran se introdujo, comercialmente, en 1965 y la OMS lo clasifica como altamente peligrosos (Clase lb; LD50 en ratas $=8 \mathrm{mg} / \mathrm{kg}$ ). ${ }^{17} \mathrm{En}$ otros países de América Latina se han identificado otros productos similares que contienen aldicarb, como el denominado Tres Pasitos. ${ }^{1-7,12,13,15}$ La estimulación excesiva de los receptores de acetilcolina y, por lo tanto, el efecto excesivo sobre las 
glándulas (salivales, sudoríparas) y órganos (músculo liso, intestino y vejiga) es la hipersecreción o hiperestimulación, con manifestaciones nicotínicas (norepinefrina efecto alfa: vasoconstricción, aumento del tono del esfinter vesical, midriasis, diaforesis. Norepinefrina efecto beta: taquicardia, vasodilatación, broncoconstricción, hipotermia, leucocitosis, elevación de cuerpos cetónicos) y muscarínicas, que fueron las predominantes en el caso (miosis, lagrimeo, sialiorrea, incremento peristaltismo, leucopenia, y las "B asesinas": broncorrea, bradicardia, broncoespasmo, bradiarritmia) (Cuadro 4). ${ }^{1-7,12,13,15}$

La búsqueda electrónica de imágenes compatibles con el tóxico sin etiqueta puede ser una herramienta útil; sin embargo, no es inequívo$\mathrm{ca}$, pues un rodenticida puede ser camuflado a voluntad de un vendedor clandestino. Otros buscadores de información toxicológica incluyen TOXNET-toxicology data network en http:// toxnet.nlm.nib.gov; BUSCATOX-Área de Toxicología. Universidad de Sevilla en http://www. farmacia.us.es/toxicologia.htm ${ }^{1-15}$ así como las fichas técnicas del probable tóxico y integración diagnóstica y de un síndrome tóxico, cuando sea posible, constituyen la piedra angular para el diagnóstico y el tratamiento.

\section{CONCLUSIONES}

Los productos prohibidos respecto al uso, venta, distribución y almacenamiento de plaguicidas y rodenticidas aún están disponibles en el mercado nacional mexicano mediante venta clandestina, a pesar de las leyes precisas y estrictas. Sin duda, es útil para el médico conocer los elementos de la formulación de los rodenticidas ilícitos y, mejor aún, es generar campañas de concientización social para evitar su venta y consumo. La responsabilidad del médico ante un niño intoxicado no se limita a la sala de urgencias; trascender a la sociedad implica modificar conductas y se puede lograr mediante la educación hacia los cuidadores de los niños, advertirles de los riesgos letales de estos productos adquiridos por venta clandestina. Las redes sociales pueden resultar aliadas en estas campañas de compromiso social para evitar la comercialización y consumo de estas sustancias químicas tóxicas. Los pediatras tenemos la obligación de ser defensores de los niños y esto es, sin duda, es un gran desafío.

\section{REFERENCIAS}

1. Vates $C$, et al. Give me three steps. Pediatr Emerg Care. 2008;24(6):389-91. doi: 10.1097/PEC.0b013e31817bcedf

2. Poisoning by an illegally imported Chinese rodenticide containing tetramethylenedisulfotetramine. New York City, 2002., MMWR Morb Mortal Wkly Rep 2003;52(10): 199-201.

3. Mintegi S. Manual de Intoxicaciones en Pediatría. 3a ed. Madrid: Asociación Española de Pediatría, 2003.

4. Bucaretchi $F$, et al. Poisoning by illegal rodenticides containing acetylcholinesterase inhibitors (chumbinho): a prospective case series, Clinical Toxicology. 2012;50(1):44-51. https://doi.org/10.3109/15563650.2011.639715

5. Liu Q, et al. Poisoning deaths in China: type and prevalence detected at the Tongji Forensic Medical Center in Hubei. Forensic Sci Int. 2009;193(1-3):88-94. https://doi. org/10.1016/j.forsciint.2009.09.013

6. Marrs T, Karalliedde L. The toxicology of pesticides. 2th ed.. David Baker. Essentials of Toxicology for Health Protection: A Handbook for Field Professionals. Chapter 15. Oxford: Health Protection Agency, 2012.

7. Trueblood $A B$, et al. Pesticide-related hospitalizations among children and teenagers in Texas, 2004-2013. Public Health Rep. 2016;131(4):588-96. https://doi. org/10.1177/0033354916662218.

8. King $\mathrm{N}$, et al. Long-acting anticoagulant rodenticide (superwarfarin) poisoning: a review of iths historical development, epidemiology and clinical management. Transfus Med Rev. 2015;29(4):250-8. https://doi.org/10.1016/j. tmrv.2015.06.002

9. Uribe-Figueroa AF. Manual de urgencias de toxicología pediátrica. 2a ed. México: Intersistemas, 2014.

10. Marshall EF. Cholecalciferol: a unique toxicant for rodent control. 1984, Proceedings of the Eleventh Vertebrae Pest Conference (Enviromental Health and Protection Commons), Núm. 22. http://digitalcommons.unl.edu/vpc11/22.

11. Rubistein I. Antidote for bromethalin poisoning. Can Vet J. 2014;55(1):1185.

12. Sanborn $M$, et al. Identifyng and managing adverse environmental health effects: Pesticides. CMJA 2002; 166 (11): 1431-36. 
13. Agencia de Protección Ambiental de Estados Unidos. Rodenticidas. Capítulo 17. (En línea) 2015. (Citado el 23 de diciembre de 2016.). https://espanol.epa.gov/sites/ production-es/files/2015-09/documents/spch17.pdf.

14. Gunja N, et al. Management of intentional superwarfarin poisoning with long-term vitamin $\mathrm{K}$ and brodifacoum levels. Clin Toxicol (Phila). 2011;29(5):385-90. https://doi.org/10. 3109/15563650.2011.587126

15. Centers for Disease Control and Prevention (CDC). Maintained by: Office of Public Health Preparedness and Response
(OPHPR). Page last reviewed: April 8, 2013 (cited 2017, Jun 20). Available from: https://emergency.cdc.gov/agent/ strychnine/basics/facts.aspx.

16. Nelson L, et al. Aldicarb poisoning by an illicit rodenticide imported into the United Sates: Tres Pasitos. J Toxicol Clin Toxicol 2001;39(5);447-52.

17. Wade JS. Environmental Damages and Crimes. 15 Fla. J. In't L.39. 2002, available at http://scholarship.law.ufl. edu/facultypub/. 